\title{
Radial heat transport in packed beds at elevated pressures
}

\author{
R. J. Wijngaarden* and K. R. Westerterp** \\ Chemical Reaction Engineering Laboratories, Department of Chemical Engineering, Twente University, P.O. Box 217, \\ 7500 AE Enschede (Netherlands)
}

(Received May 14, 1991; in final form November 29, 1991)

\begin{abstract}
Values were measured for the effective radial heat conductivity $\lambda_{\text {eff, } r}$ and the heat transfer coefficient at the wall $\alpha_{w}$ in a packed bed. This was done for superficial velocities of $5-70 \mathrm{~cm} \mathrm{~s}^{-1}$ and at pressures from $1-10$ bar. Values for $\lambda_{\text {eff, } r}$ and $x_{w}$ were obtained by simultaneous fitting of measured axial and radial temperature profiles. The bed diameter was $5 \mathrm{~cm}$; it was filled with $6.1 \mathrm{~mm}$ Raschig rings. Nitrogen gas was used in all cases. Values could be determined with reasonable accuracy. The agreement with correlations presented in the literature is good for $\lambda_{\mathrm{ett}} \mathrm{r}$ and less so for $\alpha_{w}$. The results obtained indicate that $\lambda_{\mathrm{eff}, \mathrm{r}}$ and $\alpha_{\mathrm{w}}$ are a function of the product 'velocity times pressure' only. The correlations found can be represented by $\lambda_{\text {eff, } r} / \lambda_{\mathrm{g}}=21+0.23 \mathrm{Pe}$ with an average relative error of $4 \%$ and $\mathrm{Bi}=2.9 \mathrm{Pe}^{-0.40}$ with an average relative error of $5 \%$. The experiments covered the range $25<\mathrm{Pe}<350$. These correlations were obtained for one specific gas and one specific set-up.
\end{abstract}

\section{Introduction}

Several studies have been conducted on the effective radial heat conductivity $\lambda_{\text {eff, } r}$ in packed beds, $[1-11]$. In a recent review Tsotsas and Martin [12] described the different measuring techniques employed. The heat transfer coefficient at the wall, $\alpha_{w}$, has also been investigated. Mention must be made of Chao et al. [13], Hofmann [9] and Wellauer et al. [14]. A review of the properties $\lambda_{\text {eff, } \mathrm{r}}$ and $\alpha_{\mathrm{w}}$ has been given by Westerterp et al. [15].

There are some similarities in all the publications mentioned above. All the authors used pseudo-homogeneous models to determine the values of the heat transport properties, that is, all authors assumed that the gas phase and the solid phase have the same properties. In that case the heterogeneity of the packed bed is included in the values of $\lambda_{\text {eff, } r}$ and $\alpha_{w}$. The authors usually neglect the wall effect in packed beds, which is also included in the values of $\lambda_{\text {eff. } r}$ and $\alpha_{w}$. Several attempts have been made to include this wall effect in packed bed models [16].

To our knowledge none of the authors measured values for $\lambda_{\mathrm{eff}, \mathrm{r}}$ and $\alpha_{\mathrm{w}}$ at elevated pressures. Physical

*Present address: Koninklijke/Shell-Laboratorium, P.O. Box 3003, 1003 AA Amsterdam, Netherlands.

**Author to whom correspondence should be addressed. properties of the gas phase have been altered only by choosing different fluids (e.g. nitrogen, oxygen, air, hydrogen, helium, etc.), not by changing the pressure. Here, we will investigate the effect of elevated pressures on packed bed heat transport properties.

\section{Experimental}

Values of $\lambda_{\text {eff, } r}$ and $\alpha_{w}$ were determined as follows. A packed bed was heated at the wall by water boiling under high pressure. Cold nitrogen flowed through the bed at elevated pressures. A model was used to describe the steady state axial and radial temperature profiles in the packed bed as a function of $\lambda_{\text {eff, } r}$ and $\alpha_{w}$. These profiles were measured and by making a simultaneous fit we obtained values for $\lambda_{\text {eff, } r}$ and $\alpha_{w}$ as a function of the gas velocity and pressure. The superficial gas velocity based on the empty tube varied from 5 to $70 \mathrm{~cm} \mathrm{~s}^{-1}$ and the pressure between 1 and 10 bar.

A schematic drawing of the packed bed is given in Fig. 1. Nitrogen enters the inner tube in which the packed bed is situated (for dimensions see Table 1). Inside the jacket surrounding the packed bed is boiling water. The pressure inside the inner tube varies from 1 to 10 bar; in the jackel pressures between 1 and 40 bar can be chosen, corresponding to boiling points of water from 100 to $250^{\circ} \mathrm{C}$. The water inside the jacket can be 


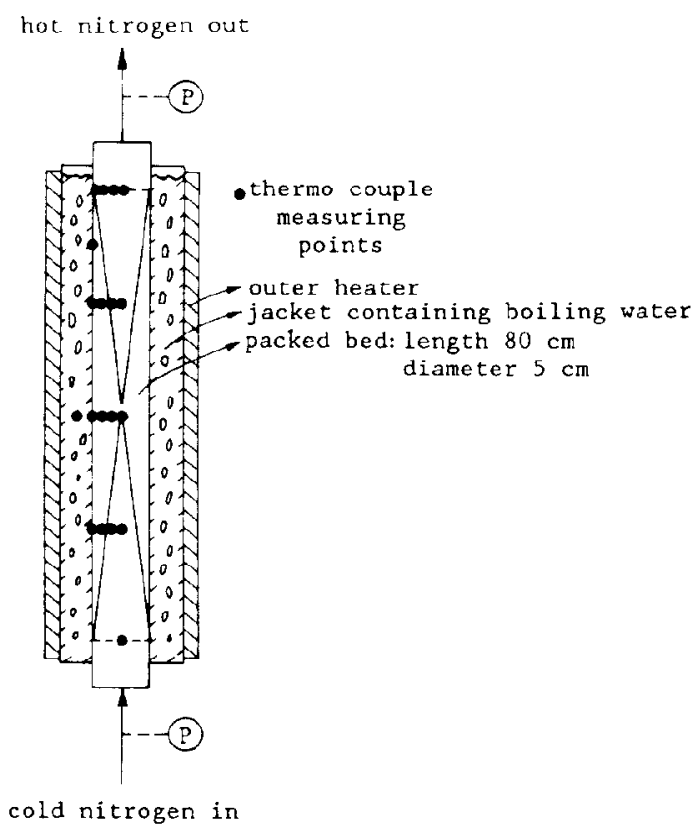

Fig. 1. The experimental set-up.

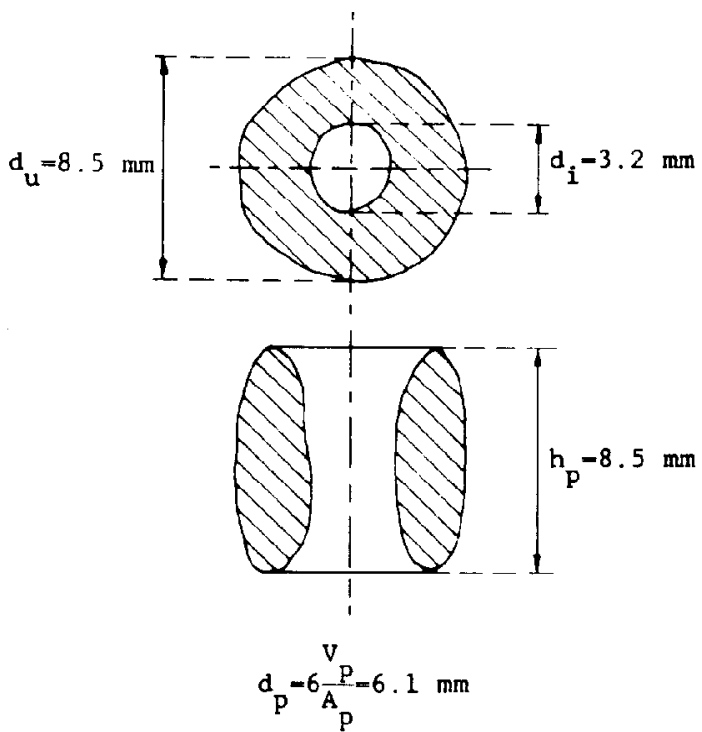

Fig. 2. The pellets used in the experiments, $h_{p}$ is the height of the particle and $d_{\mathrm{u}}$ and $d_{\mathrm{i}}$ its external and internal diameters respectively.

TABLE 1. Geometrical properties of the set-up

$$
\begin{aligned}
& d_{\mathrm{p}}=6.06 \times 10^{3} \mathrm{~m} \\
& D_{\mathrm{t}}=5.00 \times 10^{-2} \mathrm{~m} \\
& L_{\mathrm{t}}-0.800 \mathrm{~m} \\
& N_{\mathrm{D}}=8.25 \\
& \Lambda=16
\end{aligned}
$$

heated with the aid of a heater surrounding the jacket. The packed bed is made up of industrial, ring-shaped pellets (see Fig. 2).

During the experiments the pressure was monitored continuously, both at the reactor inlet and outlet. The temperature of the boiling water was measured at two locations: one at the center of the boiling liquid and the other at the wall of the inner tube. Inside the packed bed, 17 temperatures were measured (see Fig. 1). The gas inlet temperature was measured at the center of the packed bed entrance; thermocouples measured 16 other temperatures inside the packed bed at the dimensionless heights $\omega=1 / 4,1 / 2,3 / 4$ and 1 and the dimensionless radii $\rho=0,1 / 3,2 / 3$ and 1 .

\section{Theoretical}

A micro heat balance for an infinitesimally small cylindrical ring in the packed hed gives

$\frac{\partial \theta}{\partial \omega}-\frac{4 \Gamma_{\mathrm{r}}}{\operatorname{Pe} N_{\mathrm{\nu}} \Lambda} \frac{1}{\rho} \frac{\partial}{\partial \rho}\left(\rho \frac{\partial \theta}{\partial \rho}\right)=0$

In the radial direction the following boundary conditions can be applied:

$\rho=0 \Rightarrow \frac{\partial \theta}{\partial \rho}=0$
$\rho=1 \Rightarrow \frac{\partial \theta}{\partial \rho}=\frac{\mathrm{Bi} N_{\mathrm{D}}}{2}(1-\theta)$

The boundary condition in the axial direction is

$\omega=0 \Rightarrow \theta=0$

The particle diameter equivalent to a sphere $d_{\mathrm{p}}$.

$d_{\mathrm{p}}=6 V_{\mathrm{p}} / A_{\mathrm{p}}$

can be calculated from the catalyst sizes indicated in Fig. 2 and equals $6.1 \mathrm{~mm}$ for our pellets. The superficial gas velocity $v_{0}$ was measured for each experiment. The gas thermal heat conductivity and the thermal diffusivity were obtained from the Handbook of Chemistry and Physics, CRC Press, 61st edn., 1980-1981. Hence, for every experiment, the $\mathrm{Pe}$ number can be calculated. Since the values of $N_{\mathrm{D}}$ and $\Lambda$ are fixed for each experiment (see Table 1), the numbers $\Gamma_{\mathrm{r}}$ and $\mathrm{Bi}$ can be calculated.

Our results will be presented in the form of $\Gamma_{r}$ and $\mathrm{Bi}$ as a function of $\mathrm{Pe}$ and for several pressures.

\section{Results}

Here the reproducibility of the results will be discussed and an interpretation of the results given. 


\section{Reproducibility of the results}

We distinguish two sorts of reproducibility of the expcriments, the reproducibility of the heat transport properties for one specific random bed packing and the reproducibility for several bed packings. For the former we repeat the same measurement several times and in between the measurements we do not alter the bed packing. The average absolute deviation between the values measured for the heat transport properties and the corresponding mean of all values is called the reproducibility. The reproducibility for several bed packings is defined in the same way, but now between every set of experiments we altered the bed packing, that is, we emptied the inner tube and redumped the pellets.

The results, summarized in Table 2, are the average reproducibilities for a superficial gas velocity of $10 \mathrm{~cm} \mathrm{~s}^{-1}$ and ten different pressures $(1,2,3, \ldots, 9,10$ bar). At each pressure four experiments were conducted. The results indicated that neither reproducibility depended on the pressure, so both the reproducibilities were calculated from forty experiments each.

For one specific random bed packing the reproducibility of $\Gamma_{\mathrm{r}}$ and $\mathrm{Bi}$ is roughly $1 \%$ to $2 \%$. For different random bed packings the reproducibility is roughly four times the reproducibility for one specific random bed packing. This shows that the statistical character of the random packed bed is indeed significant.

\section{Interpretation of the results}

We will give an interpretation of the results and compare them with correlations from the literature. In Fig. 3, the results are plotted versus $P e$ for several pressures. The literature correlations are also indicated, the correlations recommended by Westerterp et al. [15] were used.

If we plot $\Gamma_{\mathrm{r}}$ and $\mathrm{Bi}$ versus $\mathrm{Pe}$, one single correlation is found for all the different pressures. This indicates that the numbers $\Gamma_{\mathrm{r}}$ and $\mathrm{Bi}$ indeed only depend on $\mathrm{Pe}$ (or the product $v_{0} P$ ) and not on the velocity $v_{0}$ and pressure $P$ separately. For $\Gamma_{\mathrm{r}}$ we made a best fit of the form $\Gamma_{\mathrm{r}}=A+B P e$ which yielded

$\Gamma_{\mathrm{r}}=21+0.23 \mathrm{Pe}$

TABLE 2. Reproducibility of the heat transport properties

(a) For one specific random packing

\begin{tabular}{ll}
\hline$X$ & $|\Delta X| / X$ \\
$\Gamma_{r}$ & $1.2 \%$ \\
$\mathbf{B j}$ & $1.8 \%$
\end{tabular}

(b) For different packings

$\begin{array}{ll}X & |\Delta X| / X \\ \Gamma_{r} & 7.3 \% \\ \mathrm{Bi} & 5.5 \%\end{array}$

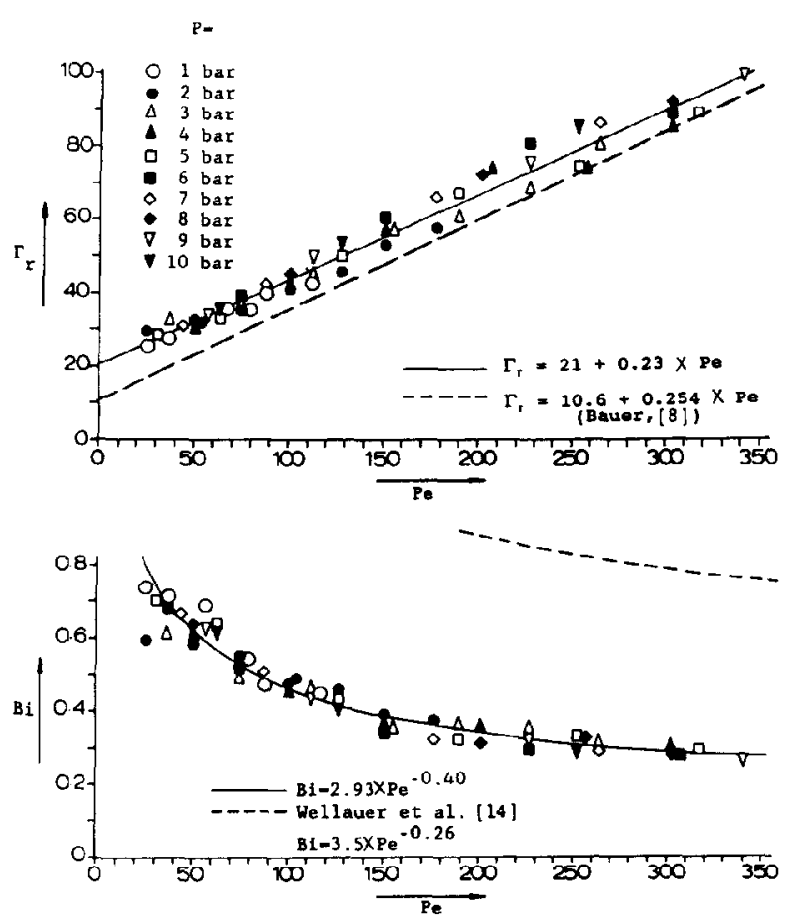

Fig. 3. The results obtained for different pressures and the correlations from the literature.

with an average relative error of $4 \%$. For the Biot number a power law $\mathrm{Bi}=A \mathrm{Pe}^{B}$ gave, as best fit,

$\mathrm{Bi}=2.9 \mathrm{Pe}^{-0.40}$

with an average relative error of $5 \%$. Hence the correlations for $\Gamma_{\mathrm{r}}$ and $\mathrm{Bi}$ are fairly accurate. The accuracy of the correlations is in agreement with the reproducibilities reported in Table 2 , roughly $1 \%$ to $2 \%$ for one bed packing and $5 \%$ to $7 \%$ for different bed packings.

Our correlations have the same form as those presented in the literature. There are, however, some discrepancies in the absolute values for $\mathrm{Bi}$. There can be several reasons for these discrepancies. Since we include the wall effect in the values of Bi we create an apparent dependence of $\mathrm{Bi}$ on the number of particles on a diameter $N_{\mathrm{D}}$. The role of the number $N_{\mathrm{D}}$ for $\Gamma_{\mathrm{r}}$ and Bi is still unclear. Zehner and Schlünder [2] do have a minor correction for the influence of $N_{\mathrm{D}}$. To our knowledge, this has not been confirmed in a detailed investigation of the role of $N_{\mathrm{D}}$ on $\Gamma_{\mathrm{r}}$ and $\mathrm{Bi}$. Since for our packed bed $N_{\mathrm{D}}$ is low, discrepancies with literature data can be expected. Finally it must be mentioned that our catalyst geometry is that of an industrial ringshaped catalyst (see Fig. 2), which has not yet been investigated. In applying correlations from the literature we have treated them as Raschig rings. In fact their geometry is somewhat in between that of a sphere and a Raschig ring. This could also, to a minor extent, 
account for the discrepancies. From the explanations given above we expect the first reason to be the most significant, and the second of minor importance.

\section{Conclusions and summary}

Values of $\Gamma_{\mathrm{r}}$ and Bi can be determined with reasonable accuracy by fitting models to measured radial and axial temperature profiles. The values determined are in good agreement with correlations reported in the literature. In our experiments $\Gamma_{\mathrm{r}}$ and $\mathrm{Bi}$ are a function only of the Pcelct number or the product $v_{0} P$. For both properties, fairly accurate correlations could be determined.

\section{Acknowledgements}

These investigations were supported (in part) by the Netherlands' Foundation for Chemical Research (SON) with the financial aid from the Netherlands' Technology Foundation (STW).

\section{Nomenclature}

$A_{\mathrm{p}} \quad$ pellet outer surface area, $\mathrm{m}^{2}$

$u_{\mathrm{g}} \quad=\lambda_{\mathrm{g}} / \mu_{\mathrm{g}} C_{\mathrm{p}, \mathrm{g}}$, thermal diffusivity of gas, $\mathrm{m}^{2} \mathrm{~s}^{-1}$

$\mathrm{Bi}=\alpha_{\mathrm{w}} d_{\mathrm{p}} / \lambda_{\mathrm{eff}, \mathrm{r}}$, Biot number for a particle

$C_{\mathrm{p}, \mathrm{g}}$ specific heat capacity of the gas, $\mathrm{J} \mathrm{kg}^{-1} \mathrm{~K}^{-1}$

$D_{\mathrm{t}} \quad$ tube diameter, $\mathrm{m}$

$d_{\mathrm{p}}=6 V_{\mathrm{p}} / A_{\mathrm{p}}$, particle diameter equivalent to a sphere, $\mathrm{m}$

$L_{\mathrm{t}} \quad$ tube length, $\mathrm{m}$

$N_{\mathrm{D}} \quad=D_{\mathrm{t}} / d_{\mathrm{p}}$, number of particles on tube diameter

$P$ pressure, $\mathrm{N} \mathrm{m}^{-2}$

$\mathrm{Pe} \quad=v_{0} d_{\mathrm{p}} / a_{\mathrm{g}}$, Peclet number

$R_{\mathrm{t}} \quad$ tube radius, $\mathrm{m}$

$r$ radial coordinate, $\mathrm{m}$

$T$ temperature, $\mathrm{K}$

$T_{0} \quad$ gas inlet temperature, $\mathrm{K}$

$T_{\mathrm{w}} \quad$ temperature of wall, $\mathrm{K}$

$V_{\mathrm{p}} \quad$ pellet volume, $\mathrm{m}^{3}$

$v_{0} \quad$ superficial velocity based on empty tube, $\mathrm{m} \mathrm{s}^{-1}$

$z \quad$ axial coordinate, $\mathrm{m}$

$\alpha_{\mathrm{w}} \quad$ heat transfer coefficient at wall, $\mathrm{W} \mathrm{m} \mathrm{m}^{-2} \mathrm{~K}^{-1}$

$\Gamma_{\mathrm{r}}=\lambda_{\text {err. } \mathrm{r}} / \lambda_{\mathrm{g}}$, dimensionless effective radial heat conductivity $\theta \quad=\left(T-T_{0}\right) /\left(T_{\mathrm{w}}-T_{0}\right)$, dimensionless temperature

$\Lambda \quad=L_{\mathrm{t}} / D_{\mathrm{t}}$, tube slenderness

$\lambda_{\text {eff, } r}$ effective radial heat conductivity, $\mathrm{W} \mathrm{m}^{-1} \mathrm{~K}^{-1}$

$\lambda_{\mathrm{g}} \quad$ heat conductivity of gas, $\mathrm{W} \mathrm{m}^{-1} \mathrm{~K}^{-1}$

$\rho \quad=r / R_{\mathrm{t}}$, dimensionless radial coordinate

$\omega \quad=z / L_{t}$, dimensionless axial coordinate

\section{References}

1 P. Zehner, Experimental and theoretical determination of the effective heat conductivity of solid beds consisting of spheres with throughflow at moderate and high temperatures, VDIForschungsh., 558 (1973) $35 \mathrm{pp}$.

2 P. Zehner and E. U. Schlünder, Effective thermal conductivity of spherical packings perfused at moderate and high temperatures, Chem.-Ing.-Tech., 45(5) (1973) 272-276.

3 F. W. Hennecke and E. U. Schlünder, Heat transfer in heated or cooled tubes with packings of spheres, cylinders and Raschig rings, Chem.-Ing.-Tech., 45(5) (1973) 277-284.

4 E. U. Schlünder, Heat and mass transfer in tubes filled with loose material, Chem.-Ing.-Tech., 38(11) (1966) 1161-1168.

5 E. U. Schlünder, Transport phenomena in packed bed reactors, ACS Symp. Ser. No. $72(1978) 110-161$.

6 J. J. Lerou and J. F. Froment, Velocity, temperature and conversion profiles in fixed bed catalytic reactors, Chem. Eng. Sri., 32 (1977) 853-861.

7 J. J. Lerou and G. F. Froment, Estimation of heat transfer parameters in packed beds from radial temperature profiles, Chem. Fing. I., 15(3) (1978) 233-237.

8 R. Bauer, Effective radial thermal conductivity of gas-permeated packed beds containing particles of different shape and size distribution, VDI-Forschungsh., 582 (1977) $39 \mathrm{pp}$.

9 H. Hofmann, Fortschritte bei der Modellierung von Festbettreaktoren, Chem.-Ing.-Tech., 51(4) (1979) 257-265.

10 D. L. Cresswell, Heat Transfor in Packed Reds, NATO ASI Ser. Ser. E, No. 110 (Chemical Reactor Design Technology), 1986, pp. $687-728$.

11 A. G. Dixon and D. I. Cresswell, Theoretical prediction of effective heat transport parameters in packed beds, AIChE J., 25(4) (1979) 663-676.

$12 \mathrm{E}$. Tsotsas and $\mathrm{H}$. Martin, Thermal conductivity of packed beds: a review, Chem. Eng. Process., 22 (1987) 19-37.

13 R. E. Chao, R. A. Cabon and M. M. Irrizarry, Wall heat transfer to chemical reactors, Can. I. Chem. Eng., 51 (1973) $67-70$.

14 T. Wellauer, D. L. Cresswell and E. L. Newson, Heat transfer in packed bed reactor tubes suitable for selective oxidation, ACS. Symp. Ser. No. 169 (1982) 527-543.

15 K. R. Westerterp, W. P. M. Van Swaaij and A. A. C. M Beenackers, Chemical Reactor Design and Operation, Wiley, Chichester, UK, 1987.

$16 \mathrm{G}$. Eigenberger and W. Ruppel, Problems of mathematical modelling of industrial fixed-hed reactors, Ger. Chem. Eng., 9 (1986) $74-83$. 